\title{
PRIMARY SUMMAND FUNCTIONS ON THREE-DIMENSIONAL COMPACT SOLVMANIFOLDS
}

\author{
CAROLYN PFEFFER
}

(Communicated by Theodore W. Gamelin)

\begin{abstract}
Leonard Richardson has shown that for a certain class of threedimensional compact solvmanifolds, projections onto $\pi$-primary summands of $L^{2}(M)$ do not preserve the continuity of functions on $M$. It is shown here that if the $\pi$-primary projection of a continuous function is $L^{\infty}$ then it is actually continuous. From this it follows that there are continuous functions on $M$ whose $\pi$-primary projections are essentially unbounded.
\end{abstract}

\section{INTRODUCTION}

Let $G$ be a solvable, connected, and simply connected Lie group, with Lie algebra $\mathbf{g}$ and cocompact discrete subgroup $\Gamma$. By a representation $\pi$ of $G$ we shall mean a strongly continuous, unitary representation of $G$ in some separable Hilbert space $H_{\pi} ; \pi$ will be called irreducible if the space $H_{\pi}$ contains no proper closed nontrivial subspace invariant under $\pi$.

Let $M$ be the space of right $\Gamma$ cosets in $G$ and give $M$ the quotient topology. There is a unique probability measure on $M$ that is invariant under the natural action of $G$ by right translation. Forming $L^{2}(M)$ with respect to this measure, we have that the action of $G$ on $M$ induces a unitary action of $G$ on $L^{2}(M): R(g) f(m)=f(m g)$ for $f \in L^{2}(M), m \in M$, and $g \in G . R$ is called the quasi-regular representation of $G$ on $L^{2}(M)$.

It is well known that $L^{2}(M)$ decomposes into the direct sum $\bigoplus H_{\pi}$, where the spaces $H_{\pi}$ are mutually orthogonal $R(G)$-invariant subspaces, and that $R$ on the space $H_{\pi}$ is a finite multiple of the irreducible representation $\pi$. [GGP, $\S$ I.2]. We let $(\Gamma \backslash G)^{\wedge}$ denote the set of irreducible representations appearing in the quasi-regular representation $R$ of $G$ on $L^{2}(M)$. Then the orthogonal projection $P_{\pi}$ of $L^{2}(M)$ onto $H_{\pi}$ is $L^{2}$-continuous and preserves $C^{\infty}(M)$ [AB, Theorem 5].

Now let $N$ be a nilpotent Lie group, connected and simply connected, with Lie algebra $\mathbf{n}$ and cocompact discrete subgroup $\Gamma$.

If the coadjoint orbits of the action of $N$ on the dual $\mathbf{n}^{*}$ are linear varieties, then $\Gamma \backslash N$ possesses the property that the orthogonal projections $P_{\pi}$ of $L^{2}(\Gamma \backslash N)$ onto $H_{\pi}$ preserve continuity [Ri1, B]. These flat-orbit nilmanifolds

Received by the editors April 30, 1991.

1991 Mathematics Subject Classification. Primary 22 E25. 
share this property with compact quotients of the three-dimensional solvable group $S_{R}$ by discrete subgroups. Here $S_{R}$ denotes the semidirect product $\mathbf{R} \propto \mathbf{R}^{2}$, where $\mathbf{R}$ acts on $\mathbf{R}^{2}$ via a one-parameter subgroup of rotations [Ril].

This work was motivated by the fact that orthogonal projections onto $\pi$ primary summands preserves continuity of functions in $L^{2}$ of both compact quotients of flat-orbit nilmanifolds and compact quotients of the group $S_{R}$. In [Ri2] Richardson proved a Fejer theorem for flat-orbit nilmanifolds. It can be shown that a similar Fejer theorem holds for compact quotients of $S_{R}$ by discrete subgroups. Let $S_{H}$ be the semidirect product $\mathbf{R} \propto \mathbf{R}^{2}$, where $\mathbf{R}$ acts upon $\mathbf{R}^{2}$ via the one-parameter subgroup $t \mapsto\left[\begin{array}{ll}\lambda^{t} & \\ \lambda^{-t}\end{array}\right]$ in $\operatorname{SL}_{2}(\mathbf{R})$, where $\lambda+\lambda^{-1}=k+1$ for some integer $k \geq 2$. Let $\Gamma$ be a cocompact discrete subgroup of $S_{H}$. It is known that orthogonal projections $P_{\pi}$ of $L^{2}\left(\Gamma \backslash S_{H}\right)$ onto $H_{\pi}$ do not preserve continuity [Ril]. It can be shown that no standard Fejer theorem exists for solvmanifolds of this type. However, the series determining the $L^{2}$ equivalence class of a projected function bears a resemblance to a standard lacunary Fourier series on $\mathbf{R} \backslash \mathbf{Z}$ (see [Z]). An adaptation of Sidon's theorem on convergence of lacunary Fourier series [Z, Theorem VI.6.1] is used to demonstrate that if the orthogonal projection $P_{\pi} f$ of a continuous function $f$ on $\Gamma \backslash S_{H}$ is an $L^{\infty}$ function, then $P_{\pi} f$ is actually continuous. Together with Theorem 3.13 in [Ri1], this implies that for each $H_{\pi}$ there is continuous $f \in L^{2}\left(\Gamma \backslash S_{H}\right)$ such that $P_{\pi} f$ is discontinuous and essentially unbounded.

\section{PreliminARIES}

Let $G$ be a connected, simply connected Lie group with Lie algebra $\mathbf{g}$, and let $\mathbf{g}^{*}$ be the vector space of linear functionals on $\mathbf{g}$. We define a sequence of ideals of the Lie algebra $\mathbf{g}$ by $\mathbf{g}^{(0)}=\mathbf{g}, \mathbf{g}^{(k)}=\left[\mathbf{g}^{(k-1)}, \mathbf{g}^{(k-1)}\right]$; this is called the derived series of $\mathbf{g}$, and $\mathbf{g}$ is said to be solvable if $\mathbf{g}^{(n)}=0$ for some $n \in \mathbf{N}$. We define another sequence of ideals of the Lie algebra $\mathbf{g}$ by $\mathbf{g}_{(0)}=\mathbf{g}$, $\mathbf{g}_{(k)}=\left[\mathbf{g}_{(k-1)}, \mathbf{g}\right]$; this is called the lower central series of $\mathbf{g}$, and $\mathbf{g}$ is said to be nilpotent if $\mathbf{g}_{(n)}=0$ for some $n \in \mathbf{N}$ (see [H, §3]. Throughout this paper, the term "nilmanifold" ("solvmanifold") will refer to compact spaces $\Gamma \backslash G$, where $G$ is nilpotent (solvable) and $\Gamma$ is discrete and cocompact.

The coadjoint representation of $G$ is of central importance in the representation theory of nilpotent and solvable Lie groups. The set of equivalence classes of irreducible representations of a nilpotent Lie group $G$ is naturally parametrized by the orbit space $\mathbf{g}^{*} / \mathrm{Ad}^{*} G$; this is also true for the (completely) solvable Lie groups examined in this work. This parametrization, due to A. A. Kirillov, is freely drawn upon in this work; for details, see [CG, Chapter II].

There are two three-dimensional, solvable, nonnilpotent Lie groups with cocompact discrete subgroups, the groups $S_{H}$ and $S_{R}$. Their Lie algebras are three-dimensional vector spaces spanned by the vectors $T, X$, and $Y$, where $\exp s T=(s, 0,0), \exp s X=(0, s, 0)$, and $\exp s Y=(0,0, s)$.

There are five distinct compact quotients of $S_{R}$ and infinitely many distinct compact quotients of $S_{H}$.

We have the following compact quotients of $S_{H}$, with convenient coordinatizations.

Suppose $k \in \mathbf{Z}, k \geq 2$. Define $S_{H, k}=\mathbf{R} \propto \mathbf{R}^{2}$, where $\mathbf{R}$ acts on $\mathbf{R}^{2}$ via 
the one-parameter subgroup $\sigma_{k}(t)$ in $\mathrm{SL}_{2}(\mathbf{R})$ with $\sigma_{k}(1)=\left[\begin{array}{cc}1 & 1 \\ k-1 & k\end{array}\right]$. (We will henceforth refer to the normal subgroup $\mathbf{R}^{2}$ as $N$.) Then $S_{H, k} \cong S_{H}$ for each $k$. Let $\Gamma_{H, k}=\left\{(p, m, n) \in S_{H, k} ; p, m, n \in \mathbf{Z}\right\}$; then each $\Gamma_{H, k} \backslash S_{H, k}=$ $M_{H, k}$ is a distinct compact quotient of $S_{H}$.

The coordinatizations of the solvmanifolds $S_{H, k}$ just described will be referred to as the integral coordinatizations of $S_{H, k}$. Let $A \in \mathrm{GL}_{2}(\mathbf{R})$ be such that

$$
A \sigma_{k}(t) A^{-1}=\left[\begin{array}{cc}
\lambda^{t} & 0 \\
0 & \lambda^{-t}
\end{array}\right] \quad \text { where } \lambda+\lambda^{-1}=k+1 ;
$$

if we recoordinatize $N$ so that the action of $\mathbf{R}$ on $N$ is given by this oneparameter subgroup, then $\Gamma_{H, k} \cap N=A\left(\mathbf{Z}^{2}\right)$; the nondegenerate coadjoint orbits in this case are hyperbolic cylinders of the form $x y=\lambda, \lambda \in \mathbf{R}$. The 2-torus $N \cap \Gamma_{H, k} \backslash N$ in this coordinatization will be a nonstandard torus, for all $k \geq 2$. This coordinatization of $S_{H, k}$ will be referred to as the hyperbolic coordinatization. We henceforth fix $S_{H, k}$ and refer to it as $S$ and to $\Gamma_{H, k}$ as $\Gamma$.

Consider the quasi-regular representation of $S$ on $L^{2}(M)$. Since $M$ is a compact solvmanifold, $L^{2}(M)$ decomposes canonically into the discrete direct sum of subspaces $H_{\pi}$, which are invariant with respect to the action of $S$, and are such that when the action of $S$ is restricted to the subspace $H_{\pi}, S$ acts on $H_{\pi}$ as some finite multiple of an irreducible representation $\pi$ of $S$. There exists no canonical decomposition of $H_{\pi}$ into irreducible subspaces unless $H_{\pi}$ is itself irreducible.

$(\Gamma \backslash S)^{\wedge}$ will denote the set of unitary irreducible representations of $S$ appearing in the quasi-regular representation $R$ of $S$ in $L^{2}(\Gamma \backslash S)^{\wedge}$.

$(\Gamma \backslash S)_{\infty}^{\wedge}$ will denote the set of those representations $\pi \in(\Gamma \backslash S)^{\wedge}$ that are infinite dimensional.

In the integral coordinatization of $S$, the coadjoint orbits satisfy

$$
(k-1) x^{2}+(k-1) x y-y^{2}=K,
$$

so that the orbits are saturated in the $T^{*}$-direction. We will call $\lambda$ an integral functional if $\left.\lambda\right|_{\mathbf{n}}=\alpha X^{*}+\beta Y^{*}, \alpha, \beta \in \mathbf{Z}$, and denote by $\mathscr{O}_{\pi}$ the orbit of $\lambda$ in $s^{*}$.

Fix some nonzero integral functional $\lambda \in \mathscr{O}_{\pi}$. We define the character $\chi_{\lambda}$ on the (abelian) nilradical $\mathbf{n}$ as follows: if $\left.\lambda\right|_{\mathbf{n}}=\alpha X^{*}+\beta Y^{*}$ then

$$
\chi_{\lambda}(0, r, s)=e^{2 \pi i(\alpha r+\beta s)} .
$$

In the hyperbolic coordinatization of $S$, integral functionals are elements of a nonstandard lattice obtained by transforming $\mathbf{Z}^{2}$. Let $\left\{\lambda_{i}\right\}_{i=1}^{\operatorname{mul} \pi}$ be a set of representatives of distinct $\Gamma$-orbits in the integral functionals of $\mathscr{O}_{\pi}, \lambda_{i}$ an integral functional for each $i$. Then for $f$ a continuous function on the solvmanifold, we have

$$
P_{\pi}(f)(\Gamma(t, x, y))=\sum_{i=1}^{\operatorname{mul} \pi} \sum_{n \in \mathbf{Z}} \tilde{f}_{i}(n+t) \chi_{\gamma_{k}^{*}(n) \lambda_{i}}(0, x, y)
$$

where for fixed $t, \tilde{f}_{i}(n+t)$ is the Fourier coefficient of $f$ at $\gamma_{k}^{*}(n) \lambda_{i}$ and where $\gamma_{k}(n)=A \sigma_{k}(n) A^{-1}$. 
1. A SidON THEOREM FOR PRIMARY SUMMAND FUNCTIONS IN $H_{\pi} \subset L^{2}(M)$

Suppose $S=\mathbf{R} \propto \mathbf{N}$ is coordinatized so that $\mathbf{R}$ acts on $N$ via the oneparameter subgroup $\sigma_{H}(t)=\left[\begin{array}{ll}\lambda^{t} & \\ \lambda^{-t}\end{array}\right]$. Then the coadjoint orbits will be "hyperbolic cylinders," saturated in the $t$-direction, given by the equations $x y=$ $k, k \in \mathbf{R}$. Let $\pi \in(\Gamma \backslash S)^{\wedge}$ be an infinite-dimensional representation. Let $P_{\pi}: L^{2}(\Gamma \backslash S) \rightarrow L^{2}(\Gamma \backslash S)$ be the orthogonal projection onto the $\pi$-primary summand of $L^{2} ; P_{\pi}$ does not preserve continuity of functions [Ri1]. Let $(\alpha, \beta)$ be a fixed lattice point in the coadjoint orbit $\mathscr{O}_{\pi}$, lying in the plane $\mathbf{R} X^{*}+\mathbf{R} Y^{*}$, noting that with the chosen coordinatization of $S$, the torus $N \cap \Gamma \backslash N$ will be a nonstandard torus, and so $(\alpha, \beta) \in \mathscr{O}_{\pi}$ satisfying $\chi_{(\alpha, \beta)}(N \cap \Gamma)=1$ will not have integer coordinates. We will call the set of $\left(\alpha^{\prime}, \beta^{\prime}\right)$ satisfying $\chi_{\left(\alpha^{\prime}, \beta^{\prime}\right)}(N \cap \Gamma)=1$ the lattice $\mathscr{L}^{*} \cdot \mathscr{L}^{*} \cap \mathscr{O}_{\pi}$ consists of finitely many $\Gamma$ orbits of integral points.

Theorem 1.1. $\mathscr{L}^{*} \cap \mathscr{O}_{\pi}$ is a Sidon set for each $\pi \in(\Gamma \backslash S)_{\infty}^{\wedge}$.

Proof. See [Ru, p. 127].

Corollary 1.2. Suppose $f \in P_{\pi}\left(L^{2}(M)\right)$ and $f \in L^{\infty}(M)$. Then for almost all fixed $t=t_{0}$, we have

$$
f\left(\Gamma\left(t_{0}, x, y\right)\right)=\sum_{(\alpha, \beta) \in \mathscr{O}_{\pi} \cap \mathscr{L}^{*}} f\left(t_{0}, \cdot, \cdot\right)^{\wedge}(\alpha, \beta) \chi_{(\alpha, \beta)}(x, y)
$$

absolutely and uniformly convergent to $f$.

Proof. Follows from the definition of a Sidon set.

Corollary 1.3. Suppose $\pi \in(\Gamma \backslash S)_{\infty}^{\wedge}$ and that $f \in L^{2}(\Gamma \backslash S)$ is continuous on $\Gamma \backslash S$. If $P_{\pi} f$ is $L^{\infty}$ then $P_{\pi} f$ is continuous.

Proof. Let $R=\{(t, 0,0) \in S: t \in \mathbf{R}\}$. Then $R$ is a subgroup of $S$.

Suppose $f \in L^{2}(\Gamma \backslash S)$ is continuous. Then for $(\alpha, \beta) \in \mathscr{L}^{*}$, we have

$$
f(t, \cdot, \cdot)^{\wedge}(\alpha, \beta)=\int_{T^{2}} f(t, x, y) \chi_{-(\alpha, \beta)}(x, y) d x d y
$$

continuous on $R$.

If $P_{\pi} f$ is in $L^{\infty}$ then we have

$$
\sum_{(\alpha, \beta) \in \mathscr{O}_{\pi} \cap \mathscr{L}^{*}}\left|f(t, \cdot, \cdot)^{\wedge}(\alpha, \beta)\right|<K\|f\|_{\infty}
$$

where $K$ is a constant not depending on $f$.

Since the inequality is independent of $t$, we have that

$$
P_{\pi} f=\sum_{(\alpha, \beta) \in \mathscr{O}_{\pi} \cap \mathscr{L}^{*}} f(t, \cdot, \cdot)^{\wedge}(\alpha, \beta) \chi_{-(\alpha, \beta)}(x, y)
$$

is the uniformly convergent sum of functions continuous on $S$. Since $P_{\pi} f$ also possesses left $\Gamma$-invariance, $P_{\pi} f$ is continuous on $\Gamma \backslash S$.

Corollary 1.4. There exists $f$, continuous on $M$, such that $P_{\pi} f$ is essentially unbounded. 
Proof. Example 5.3 in [Ril] shows that for each orthogonal projection $P_{\pi}$, there must exist an $f \in C(M)$ such that $P_{\pi} f$ is not continuous. By Corollary 1.3, however, $P_{\pi} f$ must then be essentially unbounded.

Corollary 1.5. If $f \in P_{\pi}\left(L^{2}(M)\right)$ is $L^{\infty}$, then for a.e. fixed $t_{0}$ we have that

$$
f\left(t_{0}, \cdot, \cdot\right): N \cap \Gamma \backslash N \rightarrow \mathbf{C}
$$

is a continuous function on $N \cap \Gamma \backslash N \cong T^{2}$.

Proof. By Corollary 1.2, we have that for $f \in P_{\pi}\left(L^{2}(\Gamma \backslash S)\right)$ essentially bounded, inequality (4) holds, for a.a. $t_{0}$.

Thus for a.a. fixed $t_{0}$,

$$
f\left(\Gamma\left(t_{0}, x, y\right)\right)=\sum f\left(t_{0}, \cdot, \cdot\right)^{\wedge}(\alpha, \beta) \chi_{(\alpha, \beta)}(x, y)
$$

is a uniformly convergent sum of functions that are continuous on $N \cap \Gamma \backslash N$ and so is itself continuous on $N \cap \Gamma \backslash N$.

\section{ACKNOWLEDGMENT}

This work formed part of my Ph.D. thesis, completed under the guidance of Leonard Richardson at Louisiana State University. I am grateful to the referee for suggestions that greatly simplified the presentation of these results.

\section{REFERENCES}

[AGH] L. Auslander, L. Green, and F. Hahn, Flows on homogeneous spaces, Ann. Math. Stud., no. 53, Princeton Univ. Press, Princeton, NJ, 1963.

[AB] L. Auslander and J. Brezin, Uniform distribution in solvmanifolds, Adv. Math. 7 (1971), 111-144.

[B] J. Brezin, Geometry and the method of Kirillov, Lecture Notes in Math., vol. 466, Springer-Verlag, New York, 1975, pp. 13-25.

[CG] L. Corwin and F. Greenleaf, Representations of nilpotent Lie groups and their applications, Part 1: Basic theory and examples, Cambridge Univ. Press, Cambridge, 1990.

[GGP] I. M. Gelfand, M. Graev, and I. Pyatetskii-Shapiro, Representation theory and automorphic functions, Saunders, Philadelphia, 1969.

[H] J. Humphreys, Introduction to Lie algebras and representation theory, Graduate Texts in Math., vol. 9, Springer-Verlag, New York, 1972.

[Ri1] L. Richardson, A class of idempotent measures on compact nilmanifolds, Acta Math. 135 (1975), 129-154.

[Ri2] _ , N-step nilpotent Lie groups with flat Kirillov orbits, Colloq. Math. 52 (1987), 285-287.

[Ru] W. Rudin, Fourier analysis on groups, Interscience, New York, 1962.

[Z] A. Zygmund, Trigonometric series, vol. 1, Cambridge Univ. Press, Cambridge, 1959.

Department of Mathematics, Rutgers University, New Brunswick, New Jfrsey 08903

Current address: Department of Mathematics, Florida State University, Boca Raton, Florida 33431

E-mail address : pfeffer@sunrise.cse.fau.edu 